\section{Medial Geniculate Nuclei}

John E. Mendoza

Department of Psychiatry and Neuroscience, Tulane Medical School and SE Louisiana Veterans Healthcare System, New Orleans, LA, USA

\section{Synonyms}

Medial geniculate body

\section{Definition}

Bilateral masses located on the caudal, inferior surface of the thalamus which serve as the relay nuclei for auditory input from the brainstem. The output of each is to the ipsilateral primary auditory cortex located in the temporal operculum (Heschl's gyrus). Although receiving input from both ears (as a result of incomplete fiber crossings in the brainstem), there is a slight preponderance of contralateral representation. The nuclei evidence some laminations which are thought to help preserve the tonotopic organization from the organ of Corti to the cortex. Because auditory input after the cochlear nuclei is bilateral, unilateral lesions affecting the medial geniculate nucleus would not be expected to present with clinical significant auditory deficits.

\section{Cross-References}

Auditory System 